\title{
Using Augmented Reality in Smart Manufacturing
}

\author{
Crina Narcisa Deac, Gicu Calin Deac, Cicerone Laurentiu Popa, \\ Mihalache Ghinea \& Costel Emil Cotet
}
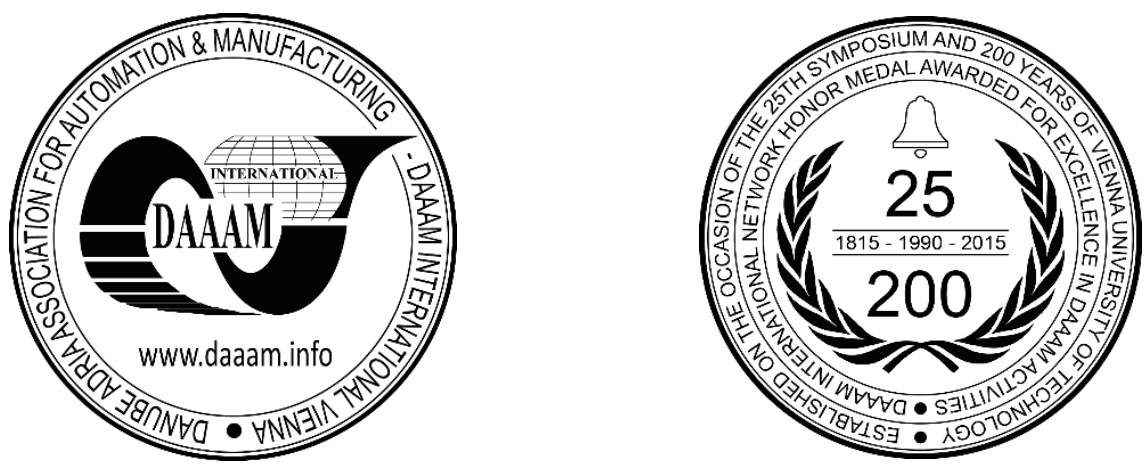

This Publication has to be referred as: Deac, C[rina] N[arcisa]; Deac, C[rina] N[arcisa]; Popa, C[icerone] L[aurentiu]; Ghinea, M[ihalache] \& Cotet, C[ostel] E[mil] (2017). Using Augmented Reality in Smart Manufacturing, Proceedings of the 28th DAAAM International Symposium, pp.0727-0732, B. Katalinic (Ed.), Published by DAAAM International, ISBN 978-3-902734-11-2, ISSN 1726-9679, Vienna, Austria

DOI: $10.2507 / 28$ th.daaam.proceedings.102

\begin{abstract}
Augmented Reality (AR) is a technology widely used in smart manufacturing, which makes data visible in real time, while the user is interacting with the real world. This paper describes a new web based AR application developed by the authors for smart manufacturing architectures, providing portability to any device, free from any existing AR platforms based on reading and interpreting markers placed on different components of industrial machines. The application provides useful real-time information to the operators: technical manuals, operating diagrams, maintenance history, components availability in the warehouse or in supplier's stock with the possibility of direct order, training videos for maintenance and work safety. Also, being connected to the smart manufacturing software and performing easier data analysis, the application is especially useful for time crucial predictive maintenance.
\end{abstract}

Keywords: smart manufacturing; predictive maintenance; augmented reality

\section{Introduction}

Recently, thanks to the emergence of the Industry 4.0, the MAS (Multi Agent Systems) combined with the new technologies (Internet of Things - IoT, wireless sensor networks, big data, cloud computing and mobile Internet, made possible the appearance of the "smart manufacturing" concept [1].

In simple terms, smart manufacturing can be considered the pursuit of data-driven manufacturing, where real-time data from sensors in the factory can be analyzed to inform decision-making. More generally, smart manufacturing can be considered a specialization of big data, whereby big data technologies and methods are extended to meet the needs of manufacturing. [2]

Industry 4.0 proposes the predictive manufacturing in the future industry. The machines are connected as a collaborative community. Such evolution requires the utilization of advance prediction tools, so that data can be systematically processed into information that can explain the uncertainties and thereby make more "informed" decisions [3]. 
The augmented reality has a broad applicability in the industry, from real-time viewing of product information and production flow[10], viewing kinematic simulations and displaying deformations and loads [9], to product design, maintenance and logistics.

Several empirical studies were conducted on the field in order to research the effectiveness of AR applied to industrial operations: J. Saaski et al. and S. J. Henderson et al. results show a performance improvement up to $50 \%$, which means that AR enables workers to perform a given task faster and with a lower possibility of error [4,5].

Following the investigations of IIoT (Industrial Internet of Things) platforms, we noticed that:

- There is no integration of predictive analytical maintenance applications with actual physical operation maintenance processes, for direct transmission and field registration of information through the operator team or autonomous robots that perform component replacement.

- There are no horizontal implementations for the availability of necessary maintenance information (components, accessories, supplies etc., or their availability to suppliers and delivery times)

- There are no integrated technical bookstores for maintenance processes (technical catalogues, procedures, etc.) that are immediately available depending on the required maintenance.

- There is no universal open bookstore for gathering and querying concrete cases of malfunctions and process parameters that led to them for easy consultation and modelling. (Through this bookstore, the cases experienced by a company can be analysed and taken into account by other companies.)

The PTC ThingWorx platform offers users the opportunity to develop VUFORIA-based AR applications, but there are no complete predefined implementations [6].

\section{Open-source mobile application for maintenance}

The research theme was proposed by FESTO Didactic and consists in building an open-source mobile application for maintenance based on WEB technologies and AR.

The purpose of this application is to provide useful information for intervention teams, such us:

- Identification of machines and their components

- State of the machinery components and subassemblies

- Information on the history of previous maintenance processes (date and time of the maintenance operation, cause, technical team etc.)

- Information on inventory of components, suppliers, prices

- Technical library (user manuals, technical manuals, labour safety etc.)

- Media library with video clips on maintenance

- Enhanced videoconference application for remote support

To implement the application, we only used web technologies, so the application can run directly into the web browser, on any device or operating system, and server-type architecture, ensuring centralization of information and ease of maintenance for libraries. The major challenge was to identify existing web technologies that could support this deployment.

We started from the basic principle of building an augmented reality application, namely:

- Taking a video stream from a webcam

- Analysis of AR identification markers

- Generating 2D or 3D augmented content

- View video stream and augmented content

For the video stream coming from the camera, we selected WebRTC / getUserMedia, which is a new HTML5 technology that does not require the installation of any additional driver or plugin.

The interface of the application was made using HTML5, CSS and javascript and for the server application we have used PHP and MySQL.
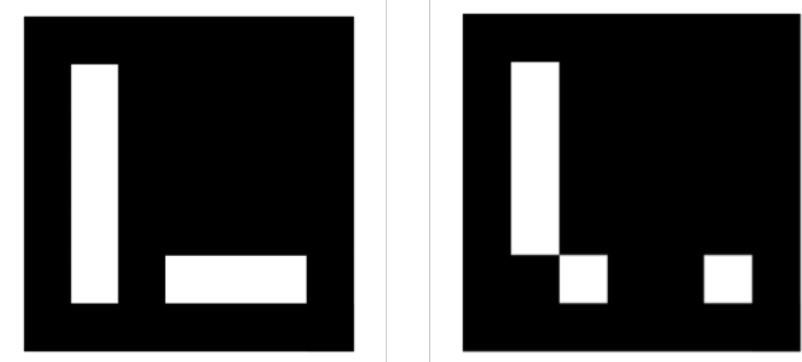

Fig. 1. Example of ArUco Markers 
For AR markers, we chose the Aruco model (figure 1.), for which there is already implementation in javascript (Aruco libraries based on OpenCV technology) [7].

For augmented 2D or 3D content we chose WebGL 2.0 technology (Web graphics library) implemented in the THREE.js library, which allows creating, importing and displaying 3D animations using Javascript without importing plugins [8].

The operating principle is as follows:

a. The operator scans by means of tablet, phone or laptop using the video camera, the markers on each machine and its components.

b. When identifying markers, the component identification information and the minimum description (image, name, serial) overlap with the image provided by the camera.

c. The operator is approaching the target component, and at that point after it is identified in the web interface, all useful information about that component is loaded.

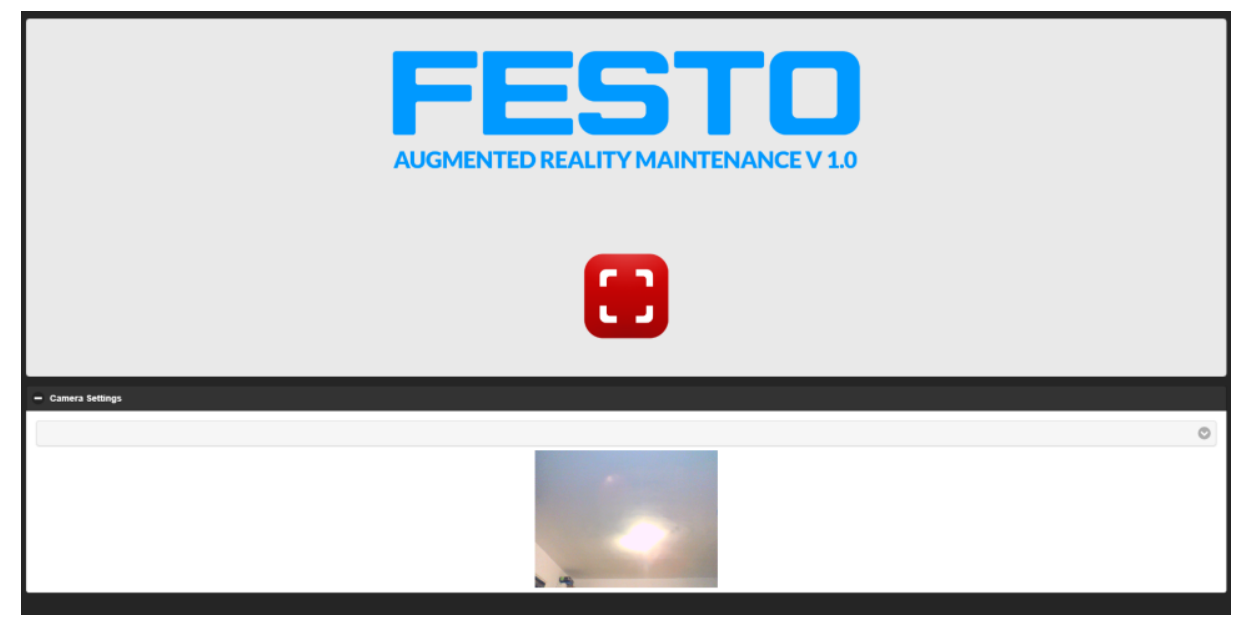

Fig. 2. Application starting screen

Once the application is accessed, the home screen is displayed and here the camcorder is selected (Camera Settings, front or rear - Figure 2). By clicking the red button, the user gets inside the Scanning screen (SCAN) where the AR application is deployed (Figure 3). The interface also contains the main menu of the application (Scan, Library, Stocks, Maintenance). The user starts the scanning. After the scan is done the software identifies the component Id and brings more information about the scanned component that can be interrogated by clicking the other items from the menu.

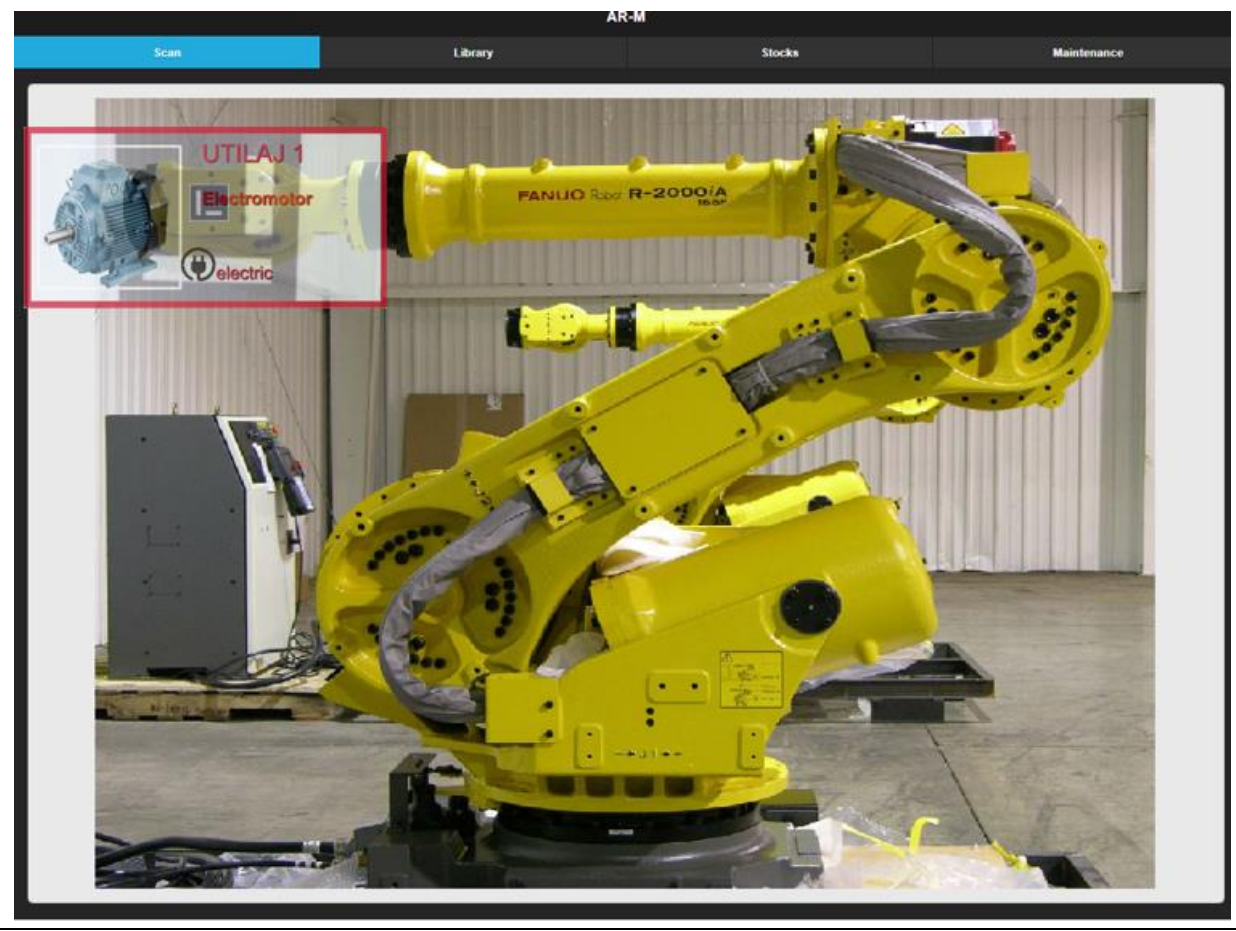


Fig. 3. Scanning page

By selecting Stocks from the main menu, an info screen (Figure 4) containing different useful information related to the scanned component is presented: general information about the component including inventory, condition, warranty and model data, purchase information, issues, existent opened tickets, info attached files and admin interface for asset edit.

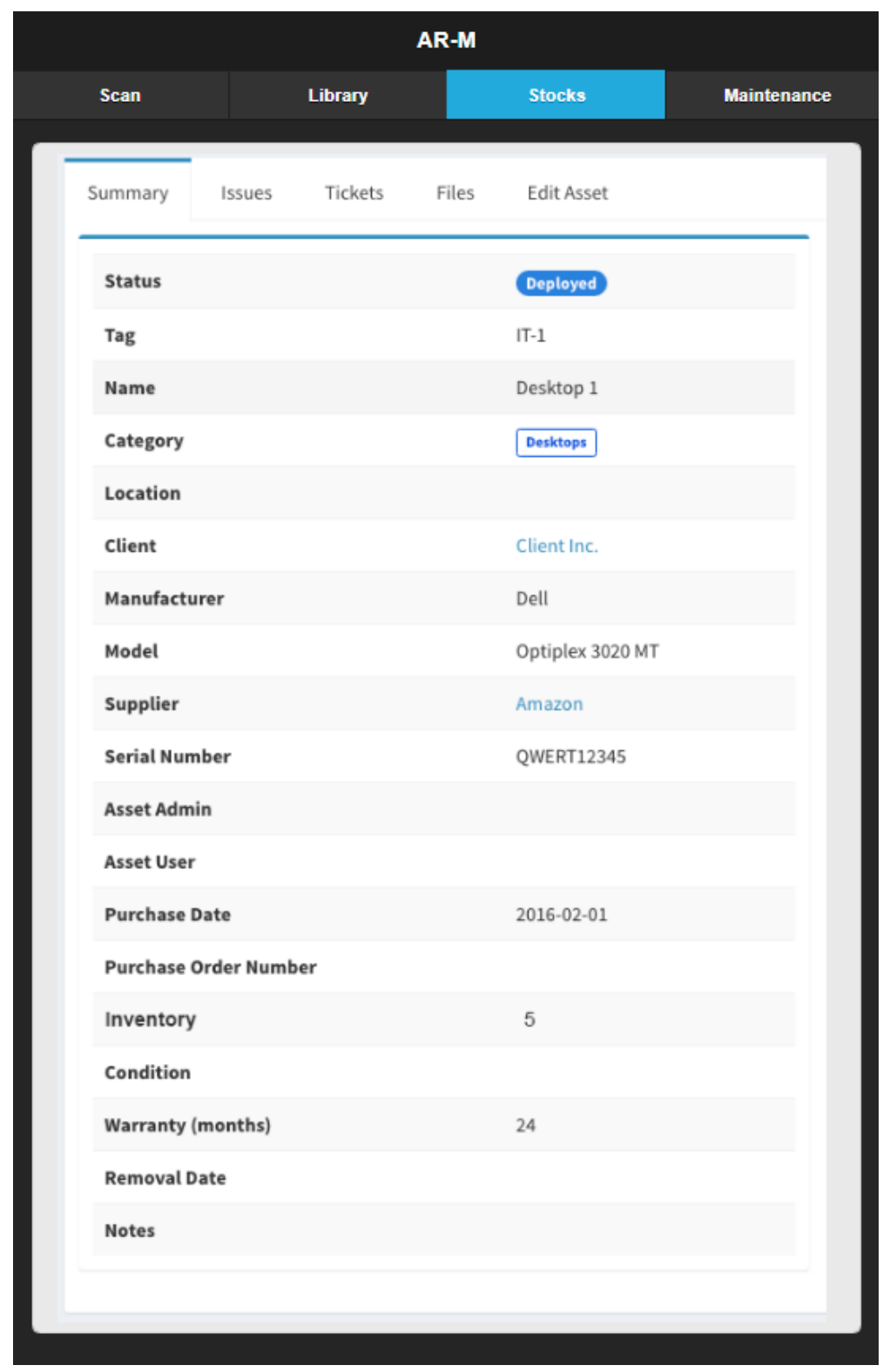

Fig. 4. Info screen

The Library menu item loads selectively, depending on the scanned AR marker, the corresponding technical library organised by visual tabs with pdf links to different articles regarding installation and maintenance or technical information about the scanned component (Figure 5).

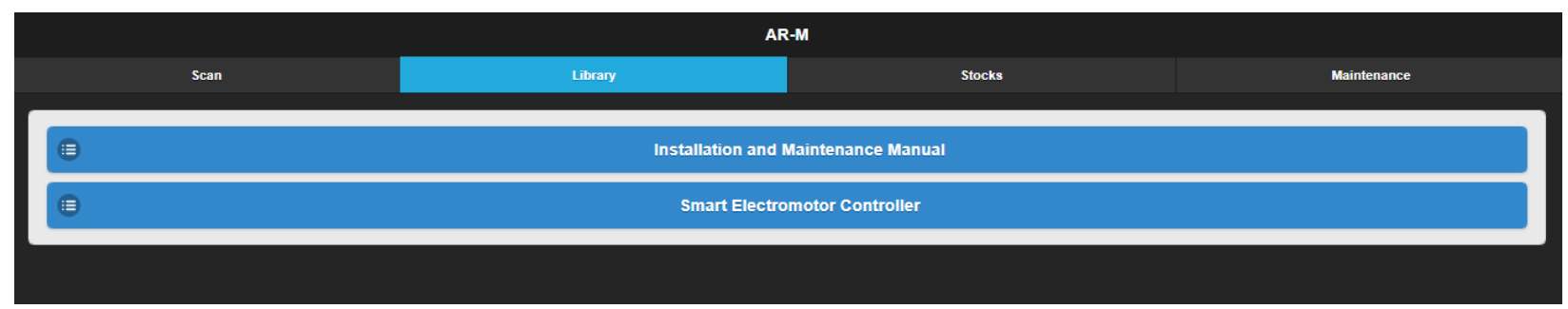




\section{Fig. 5. Technical Library}

By selecting Maintenance, the user can obtain information about the maintenance processes and history of that component, including maintenance tasks assigned to the team, statuses of the tasks and due dates, also a video library about maintenance processes and useful instructions offered by a video guide. (Figure 6)

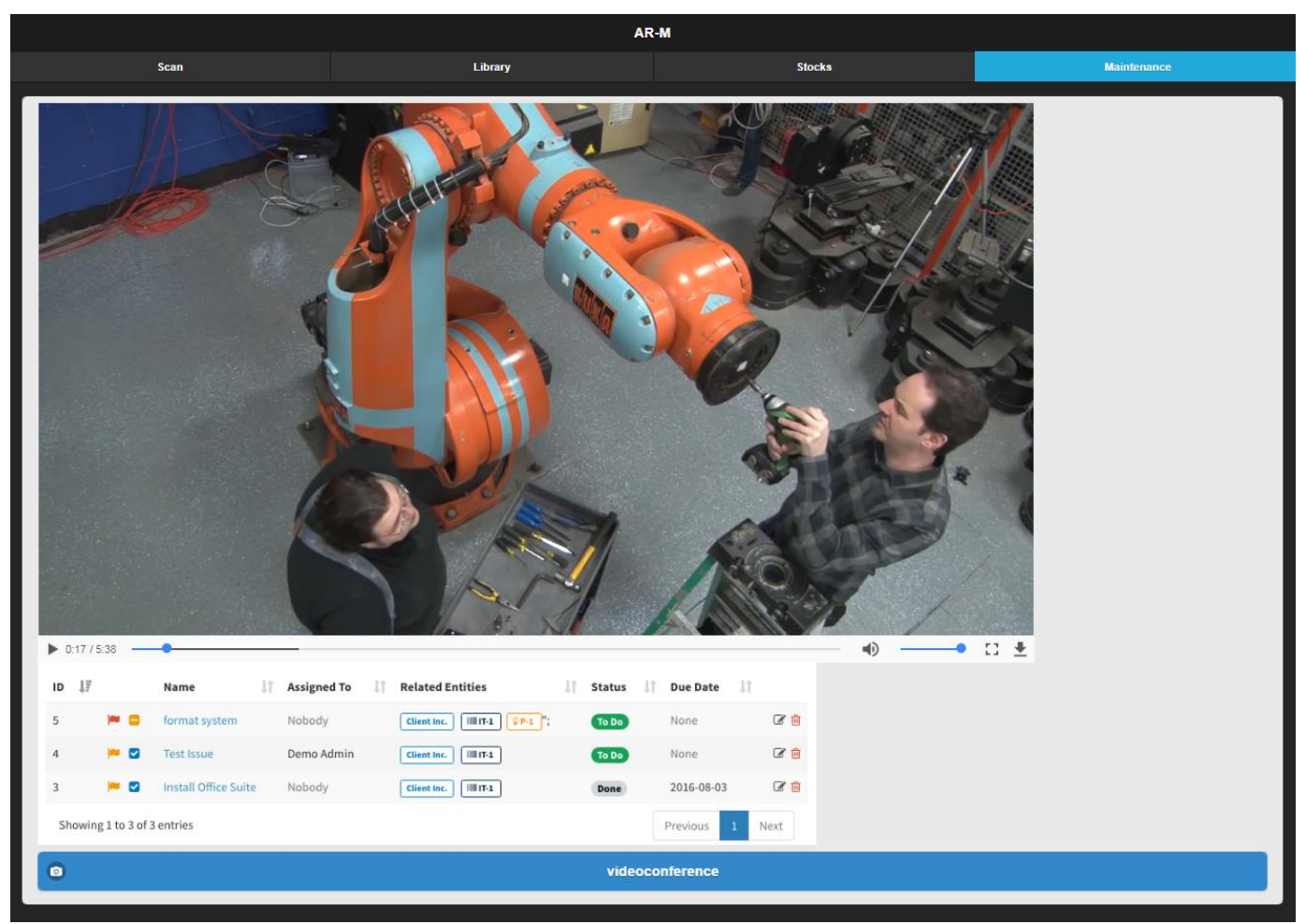

Fig. 6. Maintenance

In case the operator needs support, a Videoconference tab can be accessed and the logged user can enter a videoconference for being assisted during the maintenance process or for asking other information. Audio, video, chat, file sharing and screen sharing are the main features available inside this module (Figure 7).

The video conferencing allows simultaneous connection of multiple users and includes the following: High Definition video, audio, screen sharing, file transfer, text chat, video sharing from Youtube, SIP connection with other proprietary conference systems and allows a great remote support.

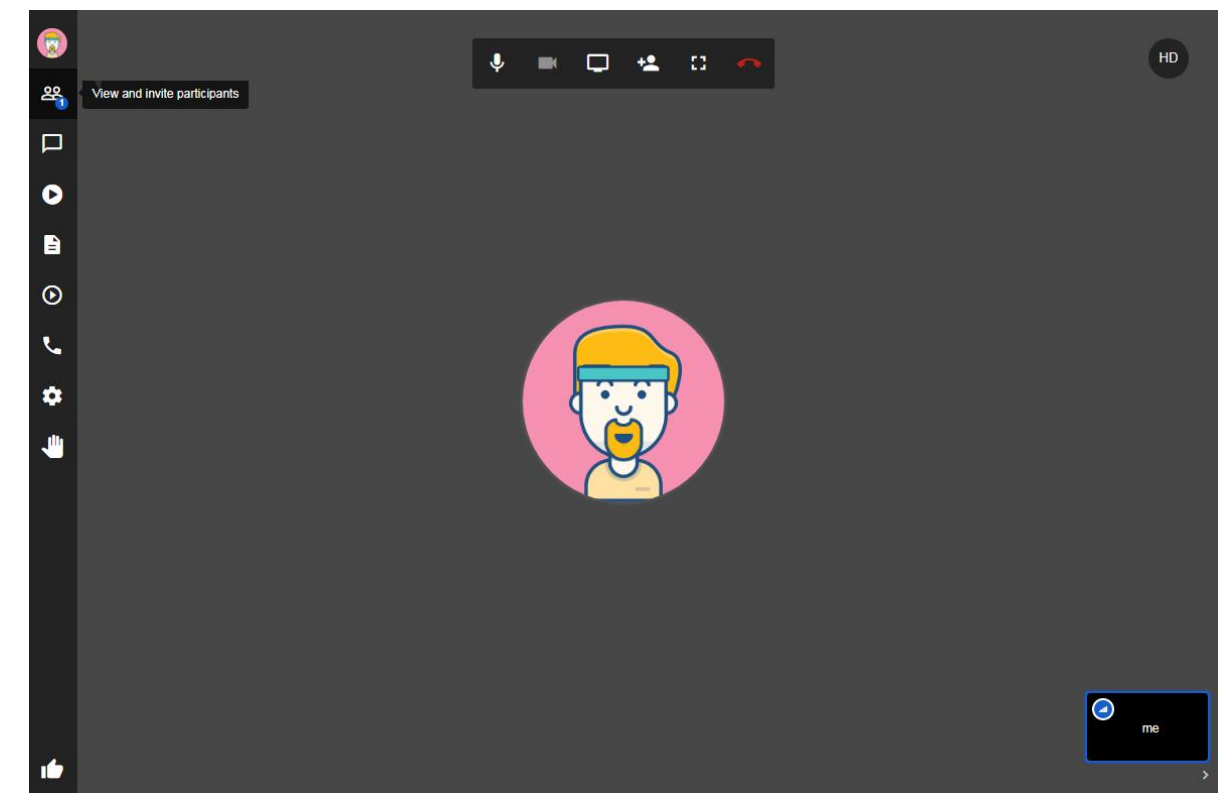

Fig. 7. AR Videoconference 


\section{Conclusion}

In our research, we have succeeded to implement an open source web AR application for maintenance which does not depend on any other proprietary cloud platform, the processing (scanning, marker identification, tracking, augmentation) is performed directly in the client's web browser using javascript, unlike other solutions requiring the installation of proprietary applications and the payment of licenses or subscriptions.

This software extends the IIoT platforms capabilities, providing useful real-time information to the operators who are on the ground and need support or immediate information: technical manuals, operating diagrams, maintenance history, components availability in the warehouse or in suppliers' stock with the possibility of direct order, training videos for maintenance and work safety.

Limitations: The only limitations of this application are that Web RTC technology used for scanning and video conferencing is not yet implemented in web browsers running on the iOS platform (iPhone, iPad) being still under implementation.

Future work: our future work will be focused on extending the mobile application for maintenance with new modules and functionalities, based on client's requests. It will contain also data processing analyze for predictive maintenance. We intend to publish online the application as an open source project.

\section{Acknowledgments}

We want to thank to our partner FESTO Didactic for collaboration and support.

\section{References}

[1] Wang, S., Wan, J., Li, D., \& Zhang, C. (2016). Implementing smart factory of industrie 4.0: An outlook. International Journal of Distributed Sensor Networks, pp. 1-10, ISSN: 15501477

[2] O’Donovan, P., Leahy, K., Bruton, K. \& O’Sullivan, D. T. J. (2015). An industrial big data pipeline for data-driven analytics maintenance applications in large-scale smart manufacturing facilities, Journal of Big Data, pp 1-26, ISSN 2196-1115

[3] Lee, J., Kao, H. A., \& Yang, S. (2014). Service innovation and smart analytics for industry 4.0 and big data environment. Procedia CIRP, pp. 3-8, ISSN: 2212-8271

[4] Sääski, J., Salonen, T., Liinasuo, M., Pakkanen, J., Vanhatalo, M., Riitahuhta, A. (2008). Augmented Reality Efficiency in Manufacturing Industry: A Case Study, Proceedings of NordDesign 2008 Conference, ISBN 9789985-59-840-5, pp. 99-109

[5] Henderson, S. J. \& Feiner, S. (2009). Evaluating the benefits of augmented reality for task localization in maintenance of an armored personnel carrier turret, 8th IEEE International Symposium on Mixed and Augmented Reality, ISBN: 978-1-4244-5390-0, pp. 135-144

[6] https://www.ptc.com/en/internet-of-things/technology-platform-thingworx, 2017, Accessed on: 2017-08-10

[7] https://sourceforge.net/projects/aruco/files, Aruco Markers for Augmented Reality applications based on OpenCV, Accessed on: 2017-08-10

[8] https://threejs.org/, Accessed on: 2017-08-10

[9] Park, H. S. \& Park, J. W.: Application Method of Augmented Reality Including FEM to Manufacturing System, Annals of DAAAM for 2011 \& Proceedings of the 22nd International DAAAM Symposium, Volume 22, No. 1, ISSN 1726-9679 ISBN 978-3-901509-83-4, Editor B. Katalinic, Published by DAAAM International, Vienna, Austria, EU, 2011

[10] Novak - Marcincin, J.; Barna, J.; Fecova, V. \& Novakova - Marcincinova, L.: Augmented Reality Applications in Manufacturing Engineering, Annals of DAAAM for 2012 \& Proceedings of the 23rd International DAAAM Symposium, Volume 23, No.1, ISSN 2304-1382, ISBN 978-3-901509-91-9, CDROM version, Ed. B. Katalinic, Published by DAAAM International, Vienna, Austria, EU, 2012 